\author{
5 Journals \\ BAHIANA

\section{Efectos de la estimulación sensorial en la conducta adaptativa del alumnado con TEA: un estudio de caso} \\ Effects of sensory stimulation on the adaptive behavior of students \\ with ASD: a case study \\ Irene Gómez-Marí1, Raúl Tárraga-Mínguez² \\ 'Autora para correspondência. Universidad de Valencia. Valencia, Comunidad Valenciana, España. \\ ORCID: 0000-0002-0452-3293. irenegomezmari@gmail.com \\ 2Universidad de Valencia. Valencia, Comunidad Valenciana, España. ORCID: 0000-0002-4458-5763. raul.tarraga@uv.es
}

RESUMEN | INTRODUCCIÓN: Las personas con trastorno del espectro autista (TEA) presentan habitualmente dificultades para procesar e integrar la estimulación sensorial de su entorno. Estas dificultades pueden verse reducidas con la intervención adecuada, especialmente en edades tempranas. El objetivo de este trabajo es evaluar los efectos que produce un programa de estimulación sensorial sobre la conducta y la inclusión educativa de un niño con TEA de educación primaria. MÉTODO: Se presenta un estudio de caso con un participante de 7 años con diagnóstico de TEA. La intervención ha constado de 52 sesiones de estimulación sensorial diseñada de acuerdo al perfil sensorial del participante. La efectividad de la intervención se ha evaluado mediante registros de observación, el Perfil sensorial Koynos, el Short Sensory Profile y el Sistema de Evaluación de Niños y Adolescentes. RESULTADOS: Los resultados de la intervención han mostrado una disminución de la frecuencia de conductas desadaptativas, lo que redunda en mejoras en los procesos de inclusión. CONCLUSIONES: El alumnado del aula ordinaria ha mostrado interés en el alumno con autismo y sus conductas desadaptativas han disminuido considerablemente. Esto sugiere que la intervención aplicada ha propiciado efectos positivos y que la estimulación sensorial es una buena solución ante los déficits de integración sensorial.

PALABRAS-CLAVE: Autismo. Conducta adaptativa. Estimulación sensorial. Inclusión.
ABSTRACT | INTRODUCTION: People with autism spectrum disorder (ASD) usually have difficulties to process and integrate the sensory stimulation of their environment. These difficulties can be reduced with adequate intervention, especially at early ages. The aim of this work is to evaluate the effects of a sensory stimulation program on the behavior and educational inclusion of a child with ASD of primary education. METHOD: A case study is presented with a 7-year-old participant diagnosed with ASD. The intervention consisted of 52 sessions of sensory stimulation designed according to the sensory profile of the participant. The effectiveness of the intervention has been evaluated through observation records, the Koynos sensory profile, the Short Sensory Profile and the Evaluation System for Children and Adolescents. RESULTS: The results of the intervention have shown a decrease in the frequency of maladaptive behaviors, what results in improvements in the inclusion processes. CONCLUSIONS: The students of the ordinary classroom have shown interest in the student with autism and his maladaptive behaviors have decreased during the intervention. That fact suggests that the intervention used has favored positive effects and that sensory stimulation is a good solution to sensory integration deficits.

KEYWORDS: Autism. Adaptive behavior. Sensory stimulation. Inclusion. 


\section{Introducción}

Yunus, Liu, Bisset y Penkala (2015) y Roley et al. (2015) estiman que alrededor del $90 \%$ de las personas con autismo presentan una integración sensorial atípica, debido a un daño en los circuitos neuronales que se dedican al procesamiento perceptivo- incluyendo las áreas sensoriales primarias. Por tanto, las respuestas que estas personas ofrecen ante los estímulos visuales, táctiles, olfativos, auditivos, propioceptivos y/o vestibulares son, según Peeters (2008), desconcertantes, resultando en ocasiones excesivas $y$ en otras, pasivas. Este hecho suele provocar una respuesta social inadecuada, que llamaremos conducta desadaptativa y que no favorece la inclusión, tal y como confirman Ferraioli y Harris (2011).

En general, las personas con desarrollo neurotípico aprenden y entienden el mundo a través de los sentidos (Demirovic et al., 2018), pues es a través de los procesos sensoriales como transformamos la información proveída por las sensaciones de nuestro cuerpo y del ambiente en mensajes con significado que nos impulsan a actuar. Erazo (2018) remarca que las personas cuya integración sensorial presenta dificultades o es desorganizada, tienen interrupciones en el procesamiento neurológico y de la información, hecho que interfiere en la habituación y sensibilización, por lo que se dice que padecen el trastorno de integración sensorial, que, según Roselló-Miranda, Berenguer-Forner y Miranda-Casas (2018), se traduce en consecuencias cognitivas, de aprendizaje, en la afectividad y conductuales, que interfieren, no solo en las relaciones personales, sino en la actividad diaria y participativa de la escuela, hecho que dificulta los procesos de inclusión.

Así, la integración sensorial fue definida por Ayres (1989) como un proceso neurológico en el que se organizan las sensaciones tanto del propio cuerpo como del entorno, permitiendo al cuerpo actuar de manera efectiva en el entorno. Ante este déficit de integrar estímulos, encontramos dos tipos de respuestassegúnlaafectaciónquepresentelapersona en ese sentido y en esa situación. Por un lado, pueden darse respuestas pasivas, en las que la percepción del input sensorial es mostrada por el alumno con una total indiferencia debido a un volumen de percepción del estímulo bajo, según Bogdashina (2007), lo que recibe el nombre de hiposensibilidad; por otro lado, pueden darse conductas descontroladas, cuando el alumno muestra hipersensibilidad denominadas por Gonthier, Longuépée y Bouvard (2016) como "reacciones exageradas ante estímulos".

Por este motivo, se hace necesaria una intervención conductual desde el ámbito educativo (Silva-Costa y Pfeifer, 2016) para propiciar la participación del alumnado, tal y como los principios de la Declaración de Salamanca (UNESCO, 1994) sostienen, dejando atrás una época en la que las personas con trastorno del espectro autista (TEA) eran consideradas como "no educables, ni moldeables" (Ferraioli y Harris, 2011). Por tanto, para favorecer no solo la presencia en el centro ordinario (Tárraga y Tarín, 2013), sino el aprendizaje y participación (Deschatelets y Poirier, 2016; Bodison y Parham, 2018) del alumnado con TEA en el ámbito educativo ordinario, es necesario conocer sus intereses y preferencias, así como modificar el entorno (Ismael, Lawson y Cow, 2015), además de estudiar su perfil sensorial.

Por ello, la intervención con el alumnado diagnosticado de TEA, será mediante la estimulación sensorial. La estimulación sensorial es, en palabras de Bodison y Parham (2018), la aplicación de estímulos de manera directa, sobre el cuerpo del niño o indirecta, con la exposición a objetos o actividades. Por este motivo, Yunus et al. (2015) perciben la estimulación sensorial como necesaria para afrontar los problemas conductuales por déficit en integración sensorial.

Peeters (2008), por su parte, matiza que la estimulación sensorial también consiste en recurrir a hábitos y rutinas que ayuden al alumnado con autismo a mantener la calma en situaciones sobrestimulatorias. Peeters (2008) enumera ciertos lugares en los que realizamos acciones predeterminadas: "Comemos en el comedor, dormimos en el dormitorio" (p.35). Por tanto, a esta serie de lugares prefijados, en los que a una actividad se le atribuye un espacio concreto se pretende añadir un espacio donde calmarse y evadirse, donde expulsar todo el malestar y ansiedad que un estímulo - una situación puede causar. 
De este modo, la estimulación sensorial ha de facilitar un clima tranquilo y positivo en el que el niño tendrá mayores oportunidades de aprendizaje (Mitchell, 2015). Así, la intervención ha de pasar por la modificación del entorno (Bodison y Parham, 2018), teniendo en cuenta las necesidades concretas del alumno, así como la dotación de material específico (Deschatelets y Poirier, 2016), como puede ser la construcción de un rincón sensorial en el aula ordinaria. Silva-Costa y Pfeifer (2016) recalcan que las actividades $\mathrm{y} / \mathrm{o}$ medidas propuestas no solo han de buscar que estas conductas desmesuradas se extingan o aminoren, sino que favorezcan la inclusión en el aula ordinaria.

De este modo, Roley et al. (2015) y Silva-Costa y Pfeifer (2016) plantean que este tipo de acciones no solo beneficiarán a unos pocos alumnos, sino al resto de niños del aula. Asimismo, en busca de la construcción de un aula ideal que atienda a todas las exigencias académicas y educativas del alumnado y persiguiendo la excelencia, la equidad, la inclusión y la armonía (Silva-Costa y Pfeifer, 2016) es importante establecer las normas y reglas del aula entre todos, para que cada alumno sea partícipe de la construcción de "contextos de actividad educativa" y facilitar así al alumno recursos equitativos, como la construcción de un rincón sensorial en el aula ordinaria o la participación del alumno en un aula multisensorial (Mitchell, 2015).

Con todo, el camino hacia la inclusión implica, no solo llevar a cabo un cambio de mentalidad, sino también cambios a nivel estructural, metodológico, y de recursos, humanos y económicos. A través de la modificación del entorno (Deschatelets y Poirier, 2016; Bodison y Parham, 2018) - siendo más pragmático y natural, más integrador, más centrado en propiciar la comunicación; en suma, más respetuoso con sus necesidades- se pueden modificar las conductas desadaptativas y que dificultan la inclusión (Ferraioli y Harris, 2011). Rivière (2010) destaca un aspecto a tener en cuenta: para que se dé una inclusión verdadera es imprescindible realizar actividades de sensibilización que involucren al claustro, a las familias, a los propios compañeros de una manera prolongada y constante, abarcando la formación y la sensibilización.
A modo de conclusión, y tal y como corroboran Tárraga y Tarín (2013), el concepto de inclusión no será adecuadamente utilizado hasta que no sirva para denominar una situación en la que el alumnado con necesidades educativas especiales (NEE), no solo esté integrado en la escuela, sino que participe de las actividades que en ella se realizan. Esta inclusión ha de darse siendo conscientes de que el autismo afecta a cada ámbito de la experiencia humana, según Robertson y Baron-Cohen (2017). Por este motivo, el objetivo del presente estudio es evaluar los efectos que produce un programa de estimulación sensorial sobre la conducta y la inclusión educativa de un niño con TEA de educación primaria.

\section{Método}

\section{Participante}

La presente intervención ha sido realizada para un alumno de 7 años (84 meses) diagnosticado, según la quinta edición del Diagnostic and Statistical Manual of Mental Disorders (DSM-5) de la American Psychiatric Association (APA, 2013), con TEA. El alumno está escolarizado bajo la modalidad específica, asistiendo a una unidad específica de comunicación y lenguaje $(C y L)$ inserta en un centro público, por lo que pasa dos sesiones diarias en el aula ordinaria de referencia que le corresponde, según su edad biológica (primer curso de educación primaria).

El alumno presenta discapacidad intelectual grave. Según el inventario de desarrollo Battelle (Newborg, Stock y Wnek, 1996) tiene un perfil cognitivo de 24-35 meses. Por lo que respecta al área de comunicación y lenguaje tiene 24-25 meses, y no presenta lenguaje funcional. Además, presenta un perfil sensorial afectado en los sentidos propioceptivo, vestibular, auditivo, táctil, gustativo y olfativo, según se comprueba en el perfil Koynos (Santamarina, 2018) y en el Short Sensory Profile (Dunn, 1999), lo cual provoca que el alumno presente numerosas conductas desadaptativas, siendo las más comunes y repetidas escupir, gritar, meterse objetos en la boca y clavar las uñas. Asimismo, según SENA FernándezPinto, Santamaría, Sánchez-Sánchez, Carrasco y del Barrio (2015), el alumno es vulnerable a ser excluido de su grupo de iguales del aula ordinaria. 


\section{Declaración ética}

La presente investigación se ha desarrollado de acuerdo a la normativa de regulación de las prácticas curriculares de la Universidad de Valencia, y se enmarca dentro de un proyecto de investigación más amplio financiado por la Agencia Estatal de Investigación de España y por la Unión Europea (EDU-2016-78867R AEI/FEDER, EU). La familia del participante ha facilitado el consentimiento para la realización del estudio.

\section{Instrumentos}

- Perfil sensorial Koynos (Santamarina, 2018). Consta de 71 ítems tipo Likert que proporcionan un perfil sensorial distinguiendo entre las modalidades sensoriales: visual, táctil, auditivo, olfativo, gustativo, propioceptivo y vestibular, e informando sobre si la afectación está relacionada con hiposensibilidad o la hipersensibilidad.

- Short Sensory Profile (Dunn, 1999). Este instrumento consta de 7 bloques con 38 ítems tipo Likert, que analizan cuatro ámbitos de la vida del alumno: sensibilidad táctil, sensibilidad del gusto y del olfato, sensibilidad kinestésica, sensibilidad visual, búsqueda de estímulos, sensibilidad auditiva y el nivel de energía-debilidad.

- Sistema de Evaluación de Niños y Adolescentes (SENA) de Fernández-Pinto et al., (2015) consta de 131 ítems Likert que evalúan las áreas que más problemas suponen para el alumno, así como las vulnerabilidades que causan en el niño malestar y que se traduce en conductas desadaptativas. También determina los recursos psicológicos que protegen al alumno ante diferentes problemas.

- Sistema de registro observacional de conductas inapropiadas y desadaptativas, es de realización propia, basado en un modelo de Carrobles y PérezPareja, (2008: 43). Tiene en cuenta la frecuencia de las conductas, los antecedentes, las consecuencias y quién está presente ante las conductas registradas.

\section{Procedimiento}

Antes de realizar la intervención, se valoraron las preferencias y gustos del sujeto durante doce sesiones en diferentes ambientes del colegio para registrar el tipo de conductas desadaptativas del sujeto, la frecuencia, el momento, los antecedentes, los consecuentes y las personas presentes cuando se da la conducta para personalizar la estimulación sensorial mediante sesiones, materiales y estrategias concretas. También fue necesario el análisis de la aplicación del perfil sensorial Koynos (Santamarina, 2018), así como del Short Sensory Profile (Dunn, 1999) y del SENA (Fernández-Pinto et al., 2015).

Tras el periodo de registro conductual, se concretaron las conductas que se querían extinguir, reducir en frecuencia $o$, simplemente, a las que se pretendía dar una alternativa socialmente aceptada. La intervención constó de un total de 33 sesiones divididas en cuatro fases (dado que una intervención en tres ámbitos distintos podía provocar una sobresaturación del alumno si no se realizaba de manera secuenciada en fases).

Esta triple intervención correspondió a la construcción de un rincón sensorial en el aula de referencia del sujeto en concreto; al uso del aula sensorial cuando el alumno estaba en la unidad CyL; y a la participación del sujeto en un taller sensorial olfativo y táctil. La intervención se realizó paulatinamente, comenzando por aquellas más presentes en su día a día (cordón y masajeador por vibración) y terminando con recursos novedosos (botella sensorial y cama elástica), todos ellos centrados, sobre todo, en la estimulación sensorial de los sentidos más afectados del sujeto: táctil, visual y vestibular. Durante la intervención, se continuó realizando el registro conductual, para estudiar su evaluación al final de la post-intervención.

Finalmente, se dedicaron siete sesiones para la elaboración de un nuevo registro conductual, las segundas aplicaciones del perfil sensorial Koynos (Santamarina, 2018), del Short Sensory Profile (Dunn, 1999), así como de la escala SENA (Fernández-Pinto et al., 2015) para analizar los efectos que la estimulación sensorial pudo tener en las conductas del sujeto, en su inclusión y en su perfil sensorial. 
La tabla 1 ofrece un resumen sistemático de los elementos esenciales de los que consta el procedimiento.

Tabla 1. Procedimiento de la intervención

\begin{tabular}{|c|c|c|c|}
\hline Fase & Descripción de la fase & Subfase & Descripción de la subfase \\
\hline $\begin{array}{c}\text { Pre-intervención } \\
\text { (del } 4 \text { al } 8 \text { de marzo) }\end{array}$ & 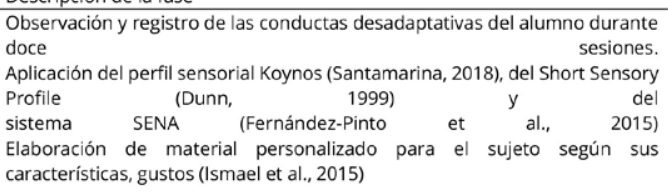 & & \\
\hline $\begin{array}{l}\text { Intervención (del } 11 \\
\text { de marzo al } 10 \text { de } \\
\text { abril) }\end{array}$ & $\begin{array}{l}\text { Intervención triple: rincón sensorial en el aula ordinaria de referencia, aula } \\
\text { sensorial en la unidad CyL y taller sensorial. La intervención es progresiva y } \\
\text { acumulativa: se van introduciendo } \quad \text { novedades. } \\
\text { Registro de las conductas desadaptativas durante } 33 \text { sesiones. }\end{array}$ & $\begin{array}{c}\text { Fase } 1 \\
\text { (del } 11 \text { al } 14 \text { de } \\
\text { marzo). } \\
\text { Fase } 2 \text { (del } 20 \text { al } \\
22 \text { de marzo) } \\
\text { Fase } 3 \\
\text { (del } 25 \text { al } 29 \text { de } \\
\text { marzo) } \\
\text { Fase } 4 \\
\text { (del } 1 \text { al } 10 \text { de } \\
\text { abril) }\end{array}$ & $\begin{array}{l}\text { En unidad CyL: introducción del alumno en el aula sensorial en } \\
\text { momentos en los que se dé un gran número seguido de conductas } \\
\text { desadaptativas. En aula ordinaria de referencia: presentación de un } \\
\text { elemento que conforma el rincón sensorial. } \\
\text { En aula ordinaria de referencia: presentación de algunos elementos } \\
\text { que conforman el rincón sensorial. } \\
\text { En aula ordinaria de referencia: presentación del rincón sensorial } \\
\text { completo } \\
\text { En aula de actividades extraescolares: inicio del taller sensorial }\end{array}$ \\
\hline $\begin{array}{c}\text { Post-intervención } \\
\text { (del } 11 \text { al } 15 \text { de } \\
\text { abril) }\end{array}$ & $\begin{array}{l}\text { Observación y registro de las conductas del sujeto durante siete sesiones. } \\
\text { Aplicación del perfil sensorial Koynos (Santamarina, 2018) y del Short } \\
\text { Sensory Profile (Dunn, 1999) por segunda ver ver ver } \\
\text { Aplicación del sistema SENA (Fernández-Pinto et al., 2015) }\end{array}$ & & \\
\hline
\end{tabular}

\section{Resultados}

Los resultados obtenidos de esta intervención surgen de los registros observacionales conductuales y anotaciones realizados a lo largo del periodo, así como de los instrumentos empleados para la obtención de información acerca de las necesidades del alumno.

\section{Resultados de la evaluación del perfil sensorial}

A continuación, se muestran dos perfiles sensoriales diferentes. La primera de las figuras representa los resultados obtenidos en la aplicación del Short Sensory Profile (Dunn, 1999) en la fase anterior y posterior a la triple intervención de estimulación sensorial. La zona sombreada (en gris) marca los resultados que una persona con integración sensorial normativa lograría. En el caso del sujeto, la primera vez que fue sometido al test (columnas en rojo) obtuvo niveles de sensibilidad afectados ("diferencia definida") en el sentido táctil, olfativo y auditivo, así como una gran necesidad de búsqueda de estímulos. En la aplicación del test en la post-intervención, se percibe una mejora notable en la sensibilidad táctil y los resultados obtenidos en la sensibilidad visual cambian de "diferencia definida" a "diferencia probable". 
Figura 1. Comparativa de las fases pre y post intervención del perfil sensorial de Dunn

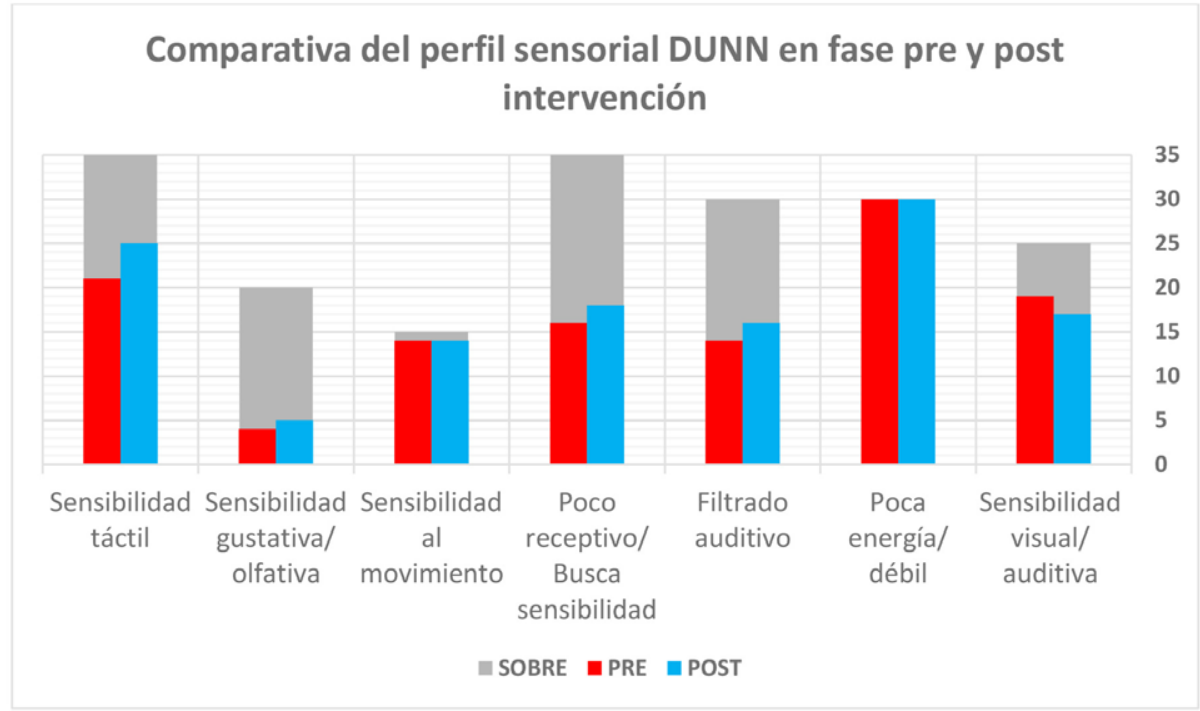

Fuente: elaboración propia.

Las figuras 2 y 3 muestran la comparación de la hipersensibilidad y la hiposensibilidad, respectivamente, en las fases ya mencionadas. Por una parte, en la figura II, se observa una leve bajada en la post-intervención de la afectación en el sentido vestibular, propioceptivo y táctil. Asimismo, ha aumentado la hipersensibilidad en estímulos gustativos y auditivos.

Figura 2. Comparativa de la hipersensibilidad de las fases pre y post intervención del perfil sensorial de Dunn

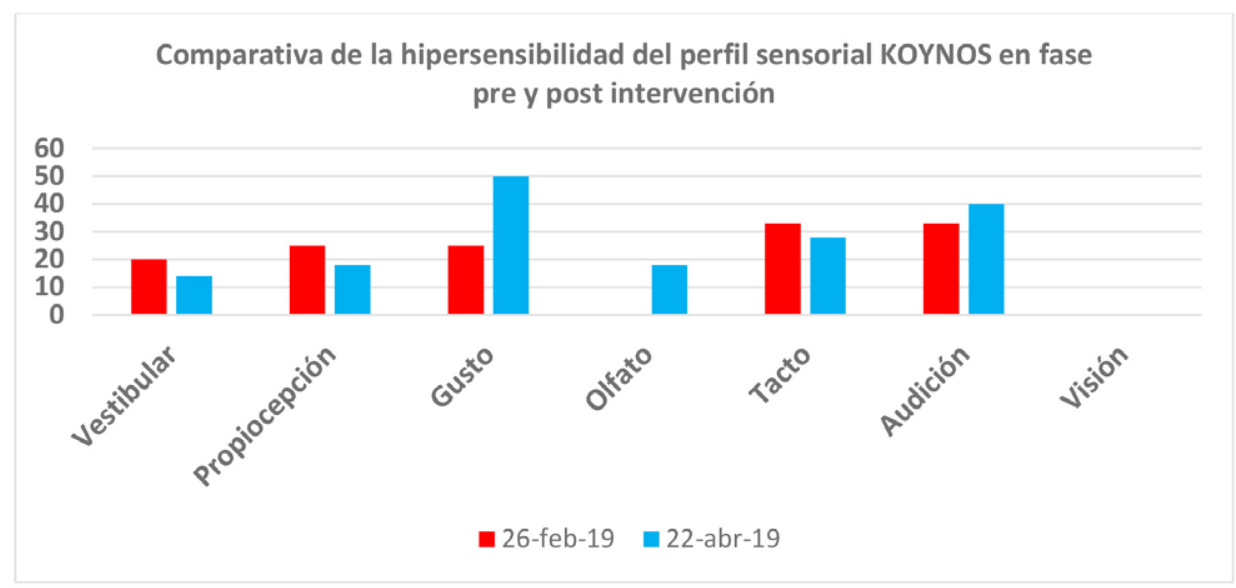

Fuente: elaboración propia.

La figura 3 muestra la evolución que ha experimentado la respuesta hiposensible del alumno ante ciertos estímulos entre la fase pre y post-intervención. La afectación de los sentidos vestibular, gustativo y táctil ha experimentado una bajada. En el caso del sentido propioceptivo y la visión, han mantenido sus valores entre ambos fases del periodo. 


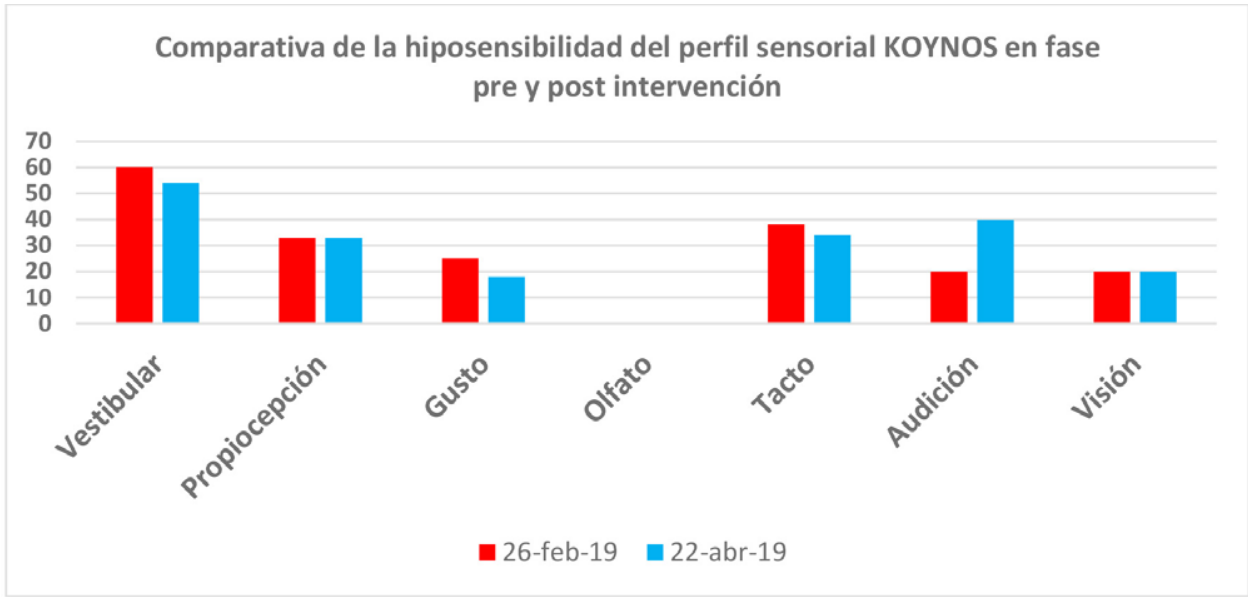

Fuente: elaboración propia.

\section{Resultados de la evaluación conductual}

La figura 4 refleja la evolución que ha experimentado el alumno en algunos de los ámbitos que contempla el instrumento SENA (Fernández-Pinto et al., 2015). Los resultados aceptables para este instrumento han de coincidir con una puntuación de 50 o una variabilidad de 10 con respecto a esta cifra. Los valores obtenidos en cuanto al índice en problemas en funciones ejecutivas (EJE), problemas de atención (ATE), problemas de hiperactividad e impulsividad (HIP), problemas de control de la ira (IRA), problemas de aprendizaje (APR), comportamientos inusuales (INU) y rigidez (RIG) disminuyen en la post-intervención con respecto a la pre-intervención.

Figura 4. Evolución de los ítems del instrumento SENA

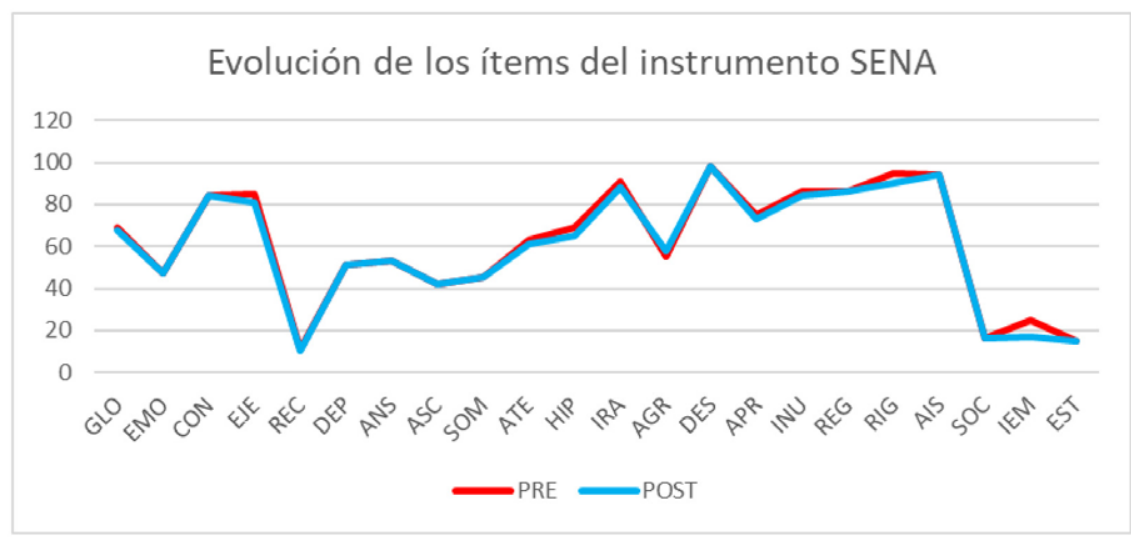

GLO: índice global de problemas; EMO: problemas emocionales; CON: problemas conductuales; EJE: problemas en funciones ejecutivas; REC: recursos personales; DEP: depresión; ANS: ansiedad; ASC: ansiedad social; SOM: quejas somáticas; ATE: problemas de atención; HIP: problemas de hiperactividad e impulsividad; IRA: problemas de control de la ira; AGR: agresión; DES: conductas desafiantes; APR: problemas de aprendizaje; INU: comportamientos inusuales; REG: problemas de regulación emocional; RIG: rigidez; AIS: aislamiento; SOC: integración y competencia social; IEM: inteligencia emocional; EST: disposición al estudio. Fuente: elaboración propia. 
A continuación, procedemos a la exposición de resultados de los datos obtenidos por el registro observacional de las cuatro conductas desadaptativas que presenta el alumno con mayor frecuencia. La figura 5 muestra cómo la conducta desadaptativa "escupe" ha disminuido notablemente a lo largo de las diferentes fases de la intervención. Es la conducta más frecuente en este sujeto.

Figura 5. Evolución de la conducta "escupe" a lo largo de las fases

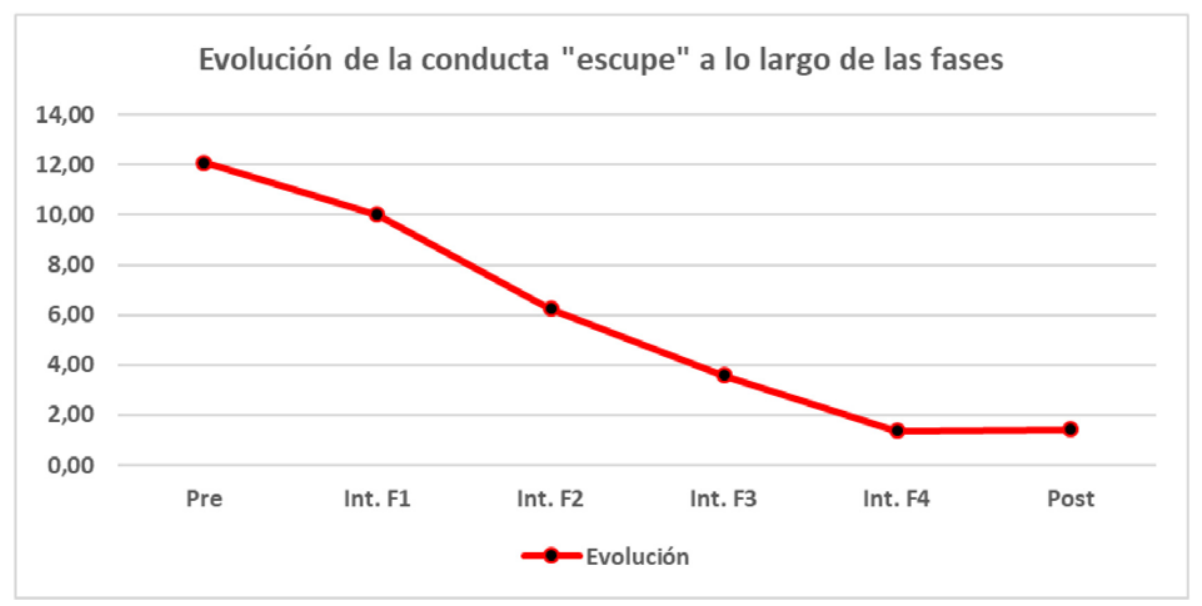

Pre: evaluación previa a la intervención; Int. F1: fase 1 de la intervención; Int. F2: fase 2 de la intervención; Int. F3: fase 3 de la intervención; Int. F4: fase 4 de la intervención; Post: evaluación posterior a la intervención. Fuente: elaboración propia.

En el caso de la evolución de la conducta "se mete objetos en la boca" también disminuye su frecuencia a lo largo de la intervención.

Figura 6. Evolución de la conducta "se mete objetos en la boca" a lo largo de las fases

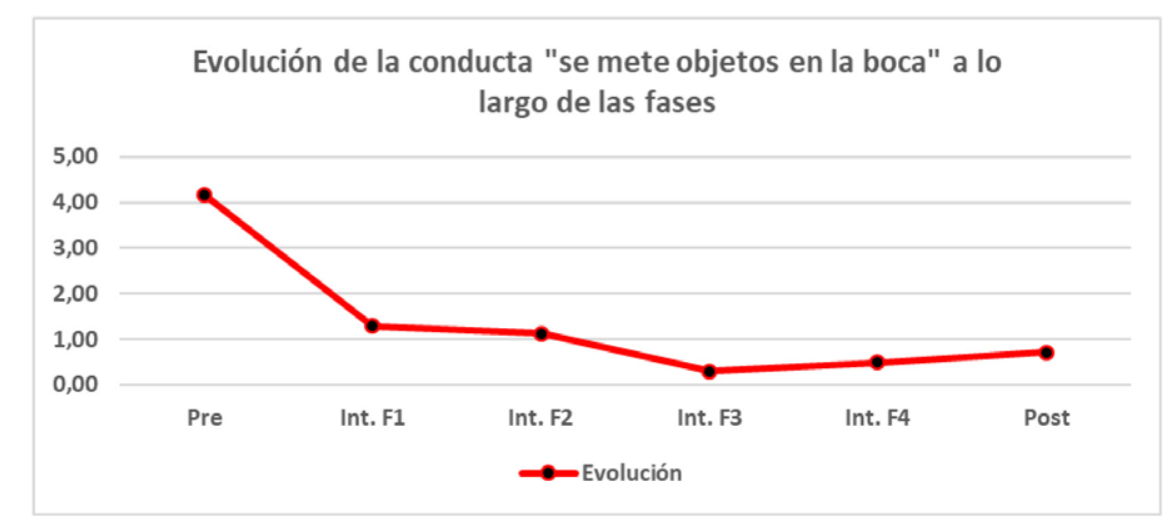

Pre: evaluación previa a la intervención; Int. F1: fase 1 de la intervención; Int. F2: fase 2 de la intervención; Int. F3: fase 3 de la intervención; Int. F4: fase 4 de la intervención; Post: evaluación posterior a la intervención. Fuente: elaboración propia.

Por lo que respecta a la conducta desadaptativa "grita", se observa una gran bajada de la pre-intervención a la primera fase de la intervención. 
Figura 7. Evolución de la conducta "grita" a lo largo de las fases

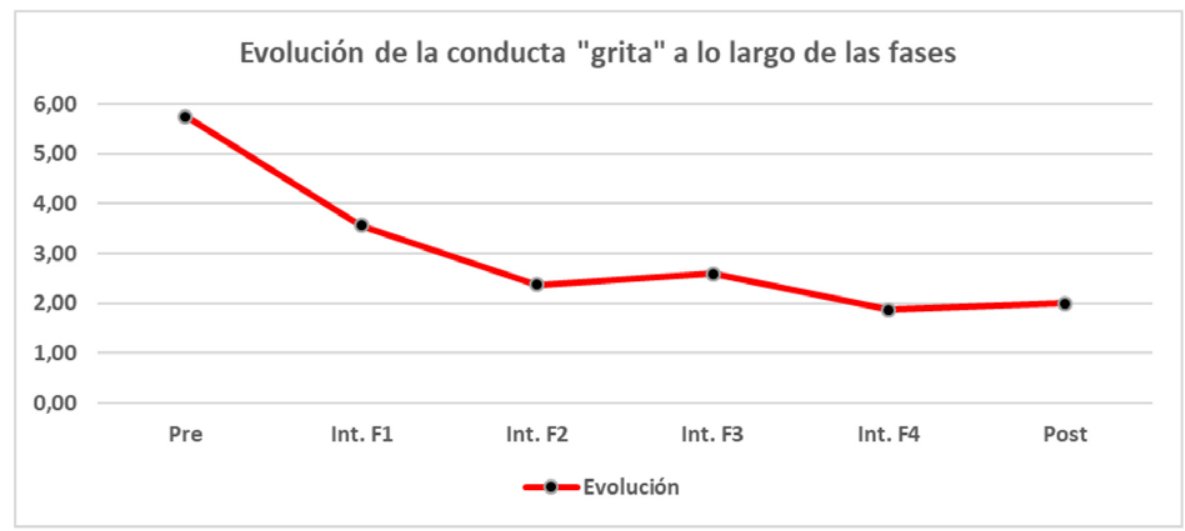

Pre: evaluación previa a la intervención; Int. F1: fase 1 de la intervención; Int. F2: fase 2 de la intervención; Int. F3: fase 3 de la intervención; Int. F4: fase 4 de la intervención; Post: evaluación posterior a la intervención. Fuente: elaboración propia.

La conducta "clava las uñas" tiene una tendencia negativa, llegando a tener una media de 0 en la fase de la postintervención. No se trata de una conducta muy frecuente, pero sí es notablemente disruptiva y muy relevante en lo que respecta a los procesos de inclusión educativa.

Figura 8. Evolución de la conducta "clava las uñas" a lo largo de las fases

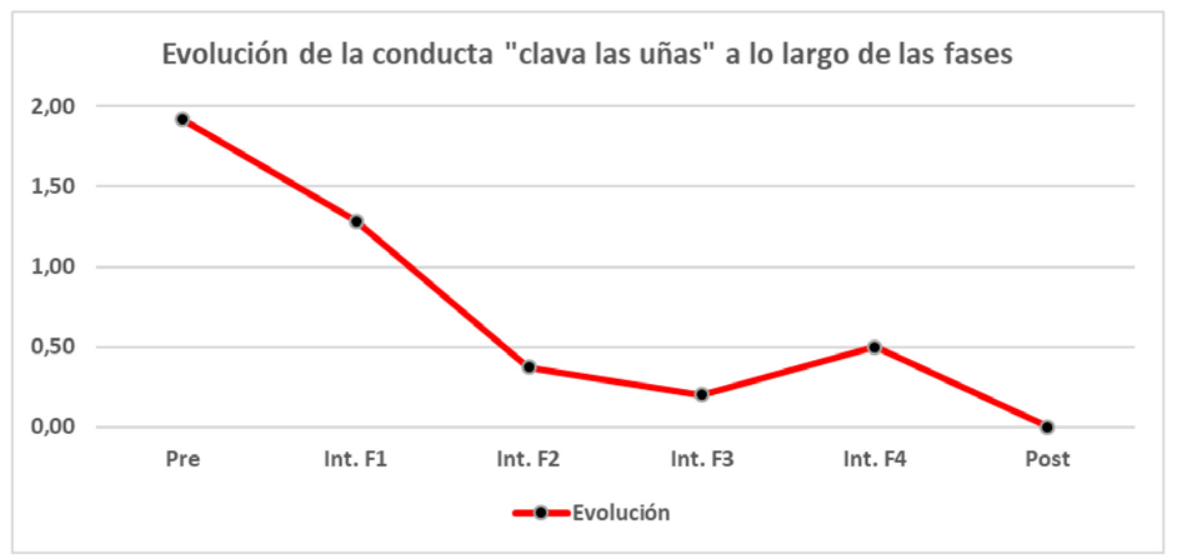

Pre: evaluación previa a la intervención; Int. F1: fase 1 de la intervención; Int. F2: fase 2 de la intervención; Int. F3: fase 3 de la intervención; Int. F4: fase 4 de la intervención; Post: evaluación posterior a la intervención. Fuente: elaboración propia.

\section{Resultados de la evaluación del riesgo de acoso escolar}

Finalmente, uno de los ítems críticos en referencia al riesgo de acoso escolar ha desaparecido en el post-test a lo largo de la intervención, tal y como observamos en la tabla II. 
Tabla 2. Comparación de la presencia de ítems de riesgo, según SENA, entre el pre y post test

\begin{tabular}{ccc}
\hline Ítems de riesgo & Aparece en & Aparece en \\
& pre-test & post-test \\
\hline
\end{tabular}

Riesgo de acoso escolar

Sus compañeros de clase le aíslan. (Siempre o casi siempre)

sí

NO

Fuente: elaboración propia.

\section{Discusión de resultados}

En el presente estudio, se percibe de manera global una leve mejoría en la frecuencia de conductas desadaptativas del sujeto, tras las sesiones de intervención de estimulación sensorial. Asimismo, el alumno muestra resultados muy positivos como una actitud más relajada, mayor sociabilidad, así como la expresión de sensación de bienestar mediante la emisión de risas y por la interacción con el adulto lo que le acerca una situación inclusiva.

En general, ambos perfiles sensoriales (Dunn, 1999; y Santamarina, 2018) sugieren una leve mejoría en el posttest. Sendos perfiles muestran una mejora en sentidos como el táctil, visual o auditivo. En el caso del Short sensory profile (Dunn, 1999) es importante destacar la mejora en la sensibilidad auditiva y visual, puesto que ha supuesto un cambio de categoría en el resultado de este test, pasando de "diferencia definida" a "diferencia probable". En el caso del sentido del gusto y el olfato, el alumno presenta una ligera mejora en la aplicación del perfil en la post-intervención.

Por su parte, en el perfil sensorial Koynos (Santamarina, 2018), destacamos en referencia a la hipersensibilidad las mejoras del sentido vestibular, propioceptivo y táctil. Estos han de ver con el propio individuo y con la interacción con el resto de personas. Por ello, una mejora en estos sentidos puede estar relacionada con una mejora en la inclusión de la persona. En cuanto a la hiposensibilidad, también experimentamos una mejora en el sistema vestibular, gustativo y táctil, coincidiendo estos dos últimos sentidos con dos de los que el Short Sensory Profile (Dunn, 1999) reevalúa favorablemente.

Por otra parte, ha habido un empeoramiento en alguno de los ítems estudiados. En particular, destacamos cómo el perfil sensorial Koynos (Santamarina, 2018) muestra que la afectación en la integración de estímulos auditivos empeora tanto hacia la hipersensibilidad como a la hiposensibilidad. Esto puede ser debido al hecho de contar con más información en la segunda aplicación del test, así como a factores externos al aula.

En el caso del sistema SENA (Fernández-Pinto et al., 2015), en la segunda aplicación del test, desapareció uno de los ítems críticos que apuntan a un posible riesgo de acoso escolar. Este hecho podría sostenerse con el incremento del interés y de la preocupación del alumnado del aula ordinaria por tratar de entender a su compañero con autismo y aprender a reaccionar ante ciertas conductas. De la misma manera, los compañeros se preocupan por el cuidado del material del rincón sensorial y entienden que no son juguetes sino instrumentos que permiten ayudarle a canalizar situaciones que provocan estrés y/o ansiedad, tanto a él como a cualquiera. 
Tal y como afirman Deschatelets y Poirier (2016), la inclusión de un alumno con autismo significa asumir desafíos tales como saber gestionar las conductas inadecuadas y la constante supervisión del mismo. Por este motivo, el hecho de aminorar la frecuencia de conductas y enseñar a autogestionarse al alumno, en este caso mediante la estimulación sensorial, supone una ventaja para los desafíos que Deschatelets y Poirier (2016) apuntaban.

Por añadidura, y tal y como queda reflejado en la figura 4, destacamos que la segunda aplicación del instrumento SENA (Fernández-Pinto et al., 2015) sugiere una leve mejoría en ítems como el Índice de problemas en las funciones ejecutivas (EJE), problemas de atención (ATE), problemas de aprendizaje (APR), comportamiento inusual (INU) y rigidez (RIG), lo que se traduce en una mejor predisposición para el trabajo, para el aprendizaje de conocimientos nuevos, para la tolerancia a la frustración ante tareas desconocidas, mayor atención y respuesta a los mandatos y mayor tolerancia a los cambios. Estas mejoras podrían deberse a la aplicación del rincón sensorial en el aula ordinaria, así como a las intervenciones en el aula sensorial y en el taller sensorial.

En cuanto a la frecuencia de conductas desadaptativas, se han registrado a lo largo del periodo comprendido entre la pre y la post-intervención un total de 611 conductas desadaptativas. El $47 \%$ de estas se ha dado en la pre-intervención. Este dato se ve reducido hasta un $5 \%$ en la post-intervención. Asimismo, esta disminución provoca una consecuencia directa en la participación e interacción del alumnado en el aula ordinaria de referencia (Roley et al., 2015).

Es interesante destacar que la tendencia de las cuatro conductas registradas (tal y como muestran las figuras $5,6,7$ y 8) es negativa, por lo que, a largo plazo, si se da continuidad a esta intervención, podrían llegar a extinguirse. De hecho, en el caso de la conducta "clava las uñas", el registro de la frecuencia llega a ser 0 en la post-intervención.
La conducta desadaptativa cuya frecuencia es mayor es "escupe", representando un 52 '7\% del total de conductas registradas. Esta coincide con ser una de las que menos favorece la inclusión y disminuye desde la etapa de la pre-intervención donde constituye un $23^{\prime} 7 \%$ de las conductas hasta el $1^{\prime} 6 \%$ en la etapa de la post-inetervención. Por ello, se podría asociar una mejora de la inclusión y de la relación social del alumno con autismo con el resto de alumnos del aula de referencia con la disminución de la frecuencia de esta conducta.

En general, cada una de las conductas presentan la bajada más acusada entre la etapa de preintervención y la primera fase de la intervención. Así, por ejemplo, la conducta "escupe" pasa de un 23'7 \% en la pre-intervención a un 11'5\% en la primera fase de la intervención; la conducta "clava las uñas" se reduce desde el 3'8\% en la pre-intervención hasta el 1'5\% en la fase 1 de la intervención. Este hecho puede ser debido a la presentación de estrategias (como el uso del cordón en el aula ordinaria o la asistencia al aula sensorial en la unidad CyL) para canalizar el malestar propiciado por el déficit de integración sensorial.

En referencia a la influencia del contexto en la aparición de conductas, observamos cómo el 47'1 $\%$ de las conductas se dan en el aula ordinaria de referencia; el $32 \%$ se dan en la unidad CyL; y el 20'9\% se dan fuera de estas dos, en ambientes como la hípica o el aula de extraescolares donde se realiza el taller sensorial. Este hecho podría ser debido a las exigencias marcadas en cada uno de estos espacios, pues en el aula de referencia, el alumno se encuentra en un contexto formal, donde hay reglas y normas muy pautadas y actividades y trabajos que requieren cierta dificultad y esfuerzo por parte del alumno; mientras que en la unidad CyL el alumno cuenta con un entorno más adecuado y preparado para sus necesidades sensoriales, aunque mantiene ciertas formalidades y normas. Sin embargo, en contextos diferentes al formal, como la hípica y durante el taller, las exigencias comportamentales sobre el alumno son menores y las dinámicas realizadas con ellos son más distendidas, por lo que el alumno se muestra más tranquilo $y$, posiblemente, presente una menor frecuencia de conductas desadaptativas. 
Por otra parte, el $12^{\prime} 3 \%$ de las conductas tiene como antecedente la presentación del trabajo que el alumno ha de hacer. Este suceso se da, sobre todo, durante las primeras etapas; sin embargo, el alumno ha ido mostrando una mayor tolerancia y predisposición al trabajo, puesto que la evolución de la frecuencia media de conductas cuyo antecedente es la presentación de una tarea ha ido disminuyendo hasta la post-intervención donde ninguna de las conductas registradas tiene este antecedente. Esta mejora también se ve reflejada en la figura 4 que representa los resultados obtenidos tras la segunda aplicación del test SENA (Fernández-Pinto et al., 2015) donde la gráfica sugiere que el alumno presenta menos problemas de aprendizaje (APR). Este hecho puede ser debido a la incorporación del rincón sensorial en momentos de trabajo y/o enseñanza-aprendizaje.

Se valora como positivo que la intervención haya sido rutinaria y variada (Bodison y Parham, 2018), puesto que el hecho de combinar diferentes modos de estimulación sensorial y diferentes ambientesel rincón, el aula y el taller- permite que el alumno establezca un seguimiento de la estrategia en diversos ambientes que le permite corregir su déficit de integración sensorial. De hecho, en los registros hay algunas evidencias de que el alumno se beneficia de la estimulación sensorial puesto que se encuentra en constante búsqueda de bienestar (Erazo, 2018), pues en diversas ocasiones pide hacer uso del columpio en el aula sensorial o demanda la voluntad de coger uno de los objetos del rincón sensorial.

Finalmente, tras el análisis de los resultados cuantitativos es necesario también hacer una interpretación cualitativa tras la intervención, a partir de las observaciones y valoraciones clínicas realizadas durante el proceso de intervención. Se puede afirmar que existe una leve mejora por lo que respecta a la tolerancia y disposición al trabajo, sobre todo en el aula de referencia, aunque también en la unidad CyL. El alumno se muestra más tranquilo en situaciones donde no hay instrucciones claras, como en los cambios de clase, así como presenta una actitud más afable con sus compañeros, permitiendo en algunos casos incluso el contacto físico con ellos. También es importante mencionar que en las observaciones de los registros a lo largo de la última etapa de la intervención y de la post-intervención aparecen conductas desadaptativas como la risa o los balbuceos. Así, aunque estas conductas sean disruptivas e interfieran en el trascurso de las sesiones del aula ordinaria, han de valorarse también como positivas, puesto que es posible que surjan porque el alumno se encuentra tranquilo, sin tensión y en calma.

\section{Limitaciones del estudio}

En referencia a las limitaciones de este estudio, destacamos la dificultad que genera que la intervención se haya tenido que ajustar al calendario escolar, por lo que se ha visto interrumpida en los períodos no lectivos. Estas dificultades del calendario han causado que, tanto la etapa de la preintervención como la de la post-intervención cuentan con un número limitado de sesiones en las que realizar registros observacionales de las conductas desadaptativas, al verse marcadas y limitadas por el tiempo del que se disponía.

La presente intervención se ha podido realizar gracias a la presencia en el aula de un segundo adulto, lo que no es una circunstancia habitual en el aula, por lo que no podemos afirmar que este tipo de intervenciones sean igualmente efectivas en los períodos en que únicamente hay un profesional en el aula. A este aspecto, concluimos que no solo hace falta ampliar el personal y disminuir las ratios, sino que estos profesionales han de abogar por la inclusión y tener formación y voluntad positiva; pues, siguiendo las conclusiones de Mitchell (2015), la inclusión educativa es un concepto multifacético que abraza una gran cantidad de factores. Así, resulta necesario informar y sensibilizar a los docentes para que sean conscientes de cuán importante es la inclusión (Mitchell, 2015), pues una de las dificultades para que esta se diera era la indiferencia del profesorado con respecto al alumno.

Asimismo, hubiese sido interesante contar con una mayor participación de las familias. Más allá de contar con su consentimiento y de informarles sobre qué, cómo y cuándo se va actuar con su hijo. Tal y como afirma Rivière (2010), sería de especial interés proponerles la construcción de un rincón sensorial en casa para que el alumno tenga una continuidad con respecto a las estrategias de estimulación sensorial antes y durante las crisis provocadas por el déficit de integración ante ciertos estímulos o por la necesidad de búsqueda de los mismos.

Finalmente, es necesario mencionar que el diseño de caso único llevado a cabo en el presente estudio no nos permite asegurar que los resultados obtenidos 
en el presente estudio sean extrapolables a otros casos de estudiantes con características similares. Los resultados del estudio apuntan efectos positivos de la intervención, pero es necesario llevar a cabo estudios con muestras de participantes más amplias para confirmar este resultado y poder afirmar que, en efecto, este tipo de intervenciones son eficaces.

\section{Futuras líneas de investigación}

De la misma manera, en el caso de que se quiera seguir profundizando en la temática de esta intervención, sería interesante tener en cuenta no solo los resultados obtenidos y las limitaciones, sino algunas recomendaciones que podrían haber hecho más precisa y rigurosa la obtención de datos. Por ejemplo, sería interesante utilizar un reloj de arena para determinar los tiempos de estimulación sensorial, con la finalidad de que el alumno entienda esta ayuda para un momento concreto en el que ciertos estímulos le sobrepasan y, por ende, su eliminación no suponga una nueva conducta desadaptativa.

Otra propuesta sería que el registro observacional contara con un apartado para registrar la duración de la conducta. Esto hubiese sido de gran interés, puesto que hay conductas que, si bien han aumentado o no se han visto disminuidas en gran medida, se ha percibido que duraban menos tiempo. Este hecho también es un indicio de mejoría a evaluar; por ello, sería recomendable realizarlo.

Para futuras líneas de investigación, podría proponerse analizar y estudiar si la estimulación sensorial tranquiliza al alumno por constituir un efecto terapéutico basado en sus intereses o, por el contrario, funciona como un premio y puede incluso aumentar las conductas desadaptativas al ser aplicada tras rabietas y/o enfados.

Por último, otra de las cosas que se ha de tener en cuentaesquesurgennuevasconductasdesadaptativas como la risa o balbuceos. Estas conductas recogidas en los registros son denominadas como conductas positivas desde el punto de vista social, emocional, relacional e interaccional del alumno; no obstante, no se ajustan a las normas comportamentales de la dinámica del grupo del aula ordinaria por lo que alejan al alumno con autismo de la inclusión.

\section{Agradecimientos}

Esta investigación se ha realizado con la financiación del Ministerio de Economía y Competitividad (EDU-2016-78867R, AEI/FEDER, UE).

\section{Contribuciones de los autores}

Gómez-Marí I y Tárraga-Mínguez R participaron en el diseño, aplicación y evaluación de la intervención descrita en el artículo, en el análisis de los resultados y en la redacción del artículo.

\section{Conflitos de intereses}

Nenhum conflito financeiro, legal o político envolvendo terceiros (gobierno, empresas y fundaciones privadas, etc.) foi declarado para nenhum aspecto do trabalho submetido (incluido más não limitando-se a subvenções e financiamentos, participación en conselho consultivo, desenho de estudo, preparación de manuscrito, análise estatística, etc.).

\section{Referencias}

American Psychiatric Association (2013). DSM-5. Diagnostic and statistical manual of mental disorders (5th Edition). Washington, DC: Author.

Ayres, A. J. (1989). Sensory integration and praxis test (SIPT). Los Angeles: Western Psychological Services.

Bodison, S. C., \& Parham, L. D. (2018). Specific sensory techniques and sensory environmental modifications for children and youth with sensory integration difficulties: A systematic review. The American Journal of Occupational Therapy, 72. doi: 10.5014/ajot.2018.029413

Bogdashina, O. (2007). Percepción sensorial en el autismo y Síndrome de Asperger. Ávila: Autismo Ávila.

Carrobles, J. A., \& Perez-Pareja, F. J. (2008). Escuela de padres: guía prácticas para evitar problemas de conducta y mejorar el desarrollo infantil. Madri: Pirámide.

Demirovic, B., Mujezinovic, A., Becarevic, M., Demirovic, N., Becarevic, N., Dizdarevic, A.,... Bratovcic, V. (2018). Tactile and hearing sensitivity of children with and without autism using the sensory profile and DSM-5. Acta Medica Saliniana, 48(2), 16-22. doi: $10.5457 / 446$

Deschatelets, J., \& Poirier, N. (2016). Accueillir un enfant présentant un trouble du spectre de l'autisme en contexte d'intervention comportamentale intensive en centre de la petite enfance. Journal on Developmental Disabilities, 22(1), 69-88. 
Dunn, W. (1999). Short Sensory Profile. United States: The Psychological Corporation.

Santander, O. A. E. (2018). Dificultades en integracion sensorial, afectividad y coducta en estudiantes de una escuela pública. Praxis y Saber, 9(20), 143-165. doi: 10.19053/22160159.v9.n20.2018.5884

Fernández-Pinto, I., Santamaría, P., Sánchez-Sánchez, F., Carrasco, M. A., \& del Barrio, V. (2015). SENA. Sistema de Evaluación de Niños y Adolescentes. Manuel de aplicación, corrección e interpretación. Madrid: TEA Ediciones

Ferraioli, S. J., \& Harris, S. L. (2011). Effective Educational Inclusion of Students on the Autism Spectrum. Journal of Contemporary Psychotherapy, 41, 19-28. doi: $10.1007 /$ s10879-010-9156-y

Gonthier, C., Longuépée, L., \& Bouvard, M. (2016). Sensory processing in low-functioningadults with autism spectrum disorder: Distinct sensory profiles and their relationships with behavioral dysfunction. Journal of Autism and Developmental Disorders, 46(9):3078-89. doi: $10.1007 /$ s10803-016-2850-1

Ismael, N. T., Lawson, L. A., \& Cox, J. A. (2015). The relationship between children's sensory processing patterns andd their leisure preferences and participation patterns. Canadian Journal of Occupational Therpy, 82(5), 316-324. doi: $10.1177 / 0008417415577421$

Mitchell, D. (2015). Inclusive Education is a Multi-Faceted Concept. CEPS Journal, 5(1), 9-30.

Newborg, J., Stock, J., \& Wnek, L. (1996). Inventario de Desarrollo Battelle. Madrid: TEA Ediciones

Peeters, T. (2008). Autismo: De la comprensión teórica a la intervención educativa. Ávila: Autismo Ávila.

Rivière, Á. (2010). Autismo. Orientaciones para la intervención educativa. Madrid: Trotta.

Robertson, C. E., \& Baron-Cohen, S. (2017). Sensory perception in autism. Nature reviews. Neuroscience, 18, 671-684. doi: 10.1038/nrn.2017.112
Roley, S. S., Mailloux, Z., Parham, L. D., Schaaf, R. C., Lane, C. J., \& Cermak, S. (2015). Sensory integration and praxis patterns in children with autism. American Journal of Occupational Thrapy, 69, 6901220010p1-p8. doi: 10.5014/ ajot.2015.012476

Roselló-Miranda, B., Berenguer-Forner, C., \& Miranda-Casas, A. (2018). Conducta adaptativa y aprendizaje en niños con trastornos del neurodesarrollo (trastorno del espectro autista y trastorno por déficit de atención/ hiperactividad). Efectos del funcionamiento ejecutivo.). Revista de neurología, 66(Supl 1), S127-S132. doi: 10.33588/ rn.66S01.2017530

Santamarina, C. (2018). Perfil sensoriak Koynos. Valencia: Koynos.

Silva-Costa, F., \& Pfeifer, L. (2016). Intervención de integración sensorial en niños con trastorno del espectro autista. Revista Chilena de Terapia Ocupacional, 16(1), 99-107.

Tárraga, R., \& Tarín, J. (2013). Escuela inclusiva: controversias en torno a discursos, políticas y actitudes. En Chisvert M., Ros A., \& Horcas V. (ed.). A propósito de la inclusión educativa: una mirada ampliada de lo escolar (1st ed., pp 28-41). Barcelona: Octaedro.

UNESCO (1994). Declaración de Salamanca y Marco de Acción para las Necesidades Educativas Especiales. Salamanca: 94/WS/18. Recuperado de: http://sid.usal.es/idocs/F8/ FDO1005/6.1.3.4-1005.pdf

Yunus, F., Liu, K., Bisset, M., \& Penkala, S. (2015). Sensory-based intervention for children with behavioral problems: A systematic review. Journal of autism and developmental disorders, 45(11), 3565-79. doi: 10.1007/s10803-015-2503-9 